\title{
Modelling of Solution Processed Indium Arsenide Nanowire Microwave Switches
}

\begin{abstract}
This paper presents the modelling of a coplanarwaveguide bottom-gated FET switch using indium-arsenide nanowires. The nanowires have been included on the switch using dielectrophoresis, which is a solution processable technique. This is a necessary first step towards developing a fully printable switch on a flexible substrate, for low cost microwave devices, built using additive manufacturing methods. The measured S-parameters show the switching capabilities of the device with an insertion loss of $9 \mathrm{~dB}$, when the switch is open (gate voltage $\geq 60 \mathrm{~V}$ ). The development of a distributed circuit model that matches the measured data is described, alongside the calculated network parameters used to represent the coplanar-waveguide and the nanowires. The model fits the measured results within $8 \%$, making it suitable for inclusion in a CAD based circuit simulator.
\end{abstract}

Index Terms - coplanar-waveguide, on-wafer measurements, nanowires, microwave switches.

\section{INTRODUCTION}

Newly emerging technologies, such as the internet of things (IoT) and the fifth generation (5G) communications have been proposing numerous applications based on wearable electronics and flexible substrates. To achieve this, a significant amount of research has been performed to fabricate various radio-frequency $(\mathrm{RF})$ and microwave devices using printing technologies [1]-[3]. Additive manufacturing techniques offer significant advantages over conventional processing, permitting rapid mask-less prototyping, generating less waste, and they are compatible with large-area, light-weight, environmentally friendly flexible substrates [4], [5].

RF switches have been used widely in phased arrays and reconfigurable areas of telecommunications systems, such as phase shifters, tunable filters and tunable antennas. PIN diodes, field-effect-transistors (FETs) and micro-electromechanical-systems (MEMS) are some of the most commonly used switching elements [6]. All of these devices are surface mountable components, which limit the potential for high density device integration and they introduce compatibility issues with flexible substrates, as they require rigid substrate materials for optimised performances [7].

In this paper, the design of a planar microwave FET switch based on a coplanar-waveguide (CPW) structure and solution processable nanowires (NWs) is presented. This is a significant step towards developing a fully printable switch, as it demonstrates the potential usage of solution processable NWs. The fabrication process used to create the switch is described, followed by measured scattering parameters. An equivalent distributed circuit model of the switch is proposed and compared with the measurement data.

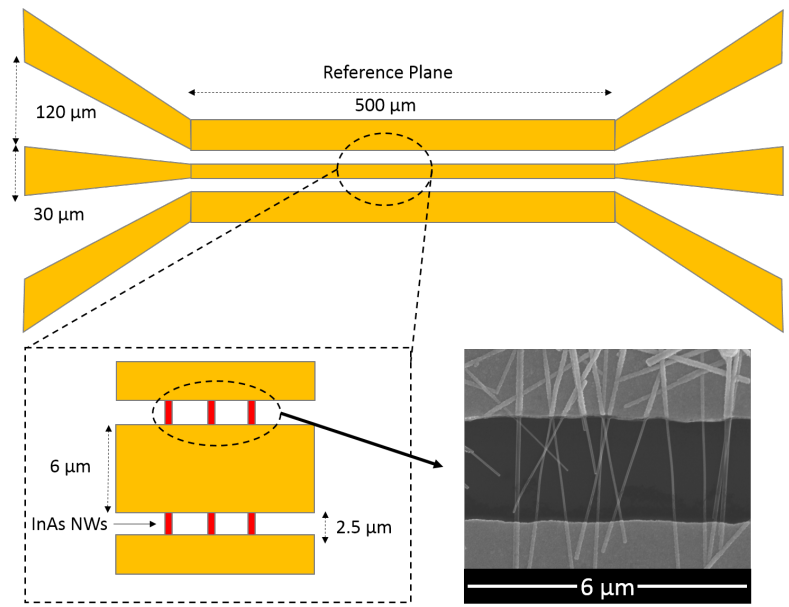

Fig. 1. Coplanar-waveguide design used for the microwave switch. The gaps of the CPW (excluding the tapered regions) are bridged with InAs NWs, forming the channel of a bottom-gated FET switch.

\section{DESIGN}

The design of the microwave switch is based on a CPW transmission line structure, as illustrated in Fig. 1. The dimensions were chosen to maintain a $50-\Omega$ characteristic impedance across the entire structure. The signal line and ground lines have a $6-\mu \mathrm{m}$ and $20-\mu \mathrm{m}$ width respectively. The gaps between the centre conductor and grounds have a width of $2.5 \mu \mathrm{m}$, which is smaller than the NWs length $(5-10 \mu \mathrm{m})$. These gaps are bridged with indium-arsenide (InAs) NWs. The conductor metal used was gold $(\mathrm{Au})$ with a $120-\mathrm{nm}$ thickness on a $500 / 1.5 \mu \mathrm{m}$ silion/silicon-dioxide $\left(\mathrm{Si} / \mathrm{SiO}_{2}\right)$ dielectric substrate. A $30 \mathrm{~nm}$ titanium (Ti) layer was used beneath the $\mathrm{Au}$ to enhance adhesion to the substrate. The NWs are placed just below the conductors, and they form the channel of a bottom-gated FET switch. Conventional microwave probes used for the physical access of planar devices, have dimensions down to $25 \mu \mathrm{m}$. This means tapered lines were required for the transition from the probe pads to the narrow CPW. The CPW switch, which is defined between the reference planes, has a 500- $\mu$ m length, as shown in Fig. 1.

\section{FABRICATION}

The InAs NWs, 50-nm in diameter, were vertically grown on an InAs substrate by metal organic vapor phased epitaxy (MOVPE) at the temperature of $597{ }^{\circ} \mathrm{C}$ with a V/III ratio of 1.6. These conditions were chosen to achieve the wurtzite crystallographic structure of the InAs NWs [8]. The substrate with the grown NWs, was sonicated in anisole to detach 


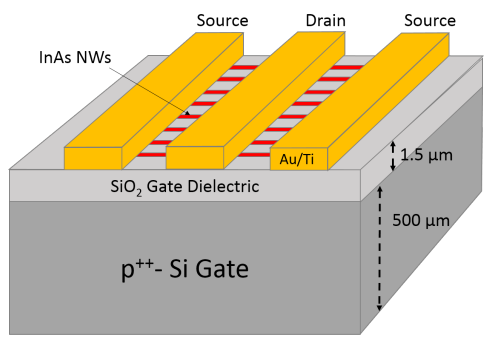

Fig. 2. Cross-section schematic of the InAs nanowire FET CPW switch.

them and form the functional InAs ink. Then, the InAs NWs were collected at the metal electrodes of interest by means of dielectrophoresis (DEP) [9]. The metal parts of the structure were fabricated by photolithography, electron-beam evaporation and lift-off techniques, including the aluminium (Al) electrodes for DEP and the Ti/Au CPW metallization that provides full metallic contact to the NWs. Prior to the evaporation of the Ti/Au metallization, the contact areas of the NWs were treated with ammonium polysulfide $\left(\left(\mathrm{NH}_{4}\right)_{2} \mathrm{~S}_{x}\right)$. This treatment removes the Al DEP electrodes and sulphur passivated the InAs surface, removing the native oxide and therefore reducing the contact resistance and making it ohmic [10]. The device structure is illustrated in Fig. 2.

\section{Measurement Set-UP}

The set-up used for the measurements of the fabricated switches is presented in Fig. 3. For the S-parameter measurements, a Keysight N5247A PNA-X was used. The frequency range of the measurements was set from 5 to $26.5 \mathrm{GHz}$, and the input power of the two ports at $-10 \mathrm{dBm}$. An on-wafer multi-line thru-reflect-line (mTRL) calibration was performed to move the reference plane of the measurement to the device (end of the tapered lines) [11]. The standards used for the calibration were a zero-length thru, an open, and two lines with lengths of $450 \mu \mathrm{m}$ and $1350 \mu \mathrm{m}$, in order to cover the desired frequency range. Two-port measurements were performed, obtaining both the reflection $\left(S_{11}\right)$ and transmission $\left(S_{21}\right)$ coefficients of the switch. In order to access the planar devices an MPI TS-2000 SE (shield environment) probe station and two MPI Titan $26 \mathrm{GHz}$ ground-signal-ground (GSG) probes with a $150 \mu \mathrm{m}$ pitch were used. The shielded environment of the probe station was purged with nitrogen to avoid the activation of the InAs NWs from moisture, since it enhances the trapping of the charge carriers by the surface states. The measurements were performed at room temperature $\left(25^{\circ} \mathrm{C}\right)$.

To locate devices on the wafer, made with a suitable number of nanowires and switch characteristics, their directcurrent (DC) measurements were at first obtained using a Keithley semiconductor parameter analyzer. The source-drain DC bias was applied to the devices using the internal bias tee of the PNA-X and through one of the two GSG probes, whereas the gate DC bias through a DC probe. Then, the S-parameters were measured at different gate bias $\left(V_{g}\right)$

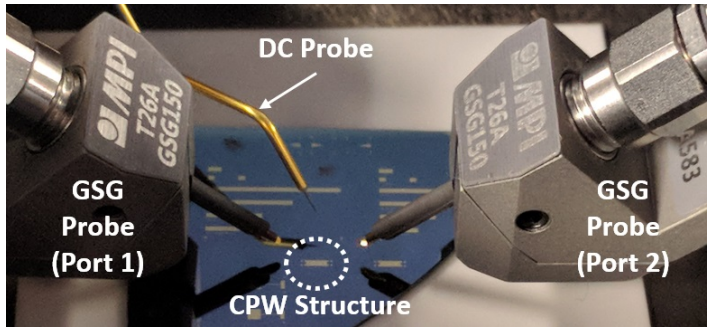

Fig. 3. Photograph of the two GSG probes and DC probe used for the excitation of the device and to provide the gate voltage respectively.

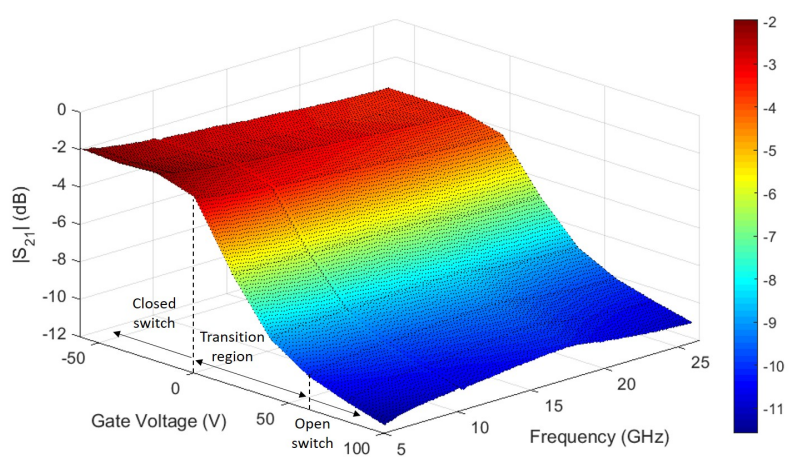

Fig. 4. Measured transmission coefficient of the device, illustrating its switching capabilities.

voltages $(-60 \mathrm{~V}$ to $+100 \mathrm{~V}$ with $20 \mathrm{~V}$ steps). The large range of the $V_{g}$ is due to the back gating of the switch.

\section{Results And Modelling}

The measured transmission coefficient of the DUT, under the different gate bias voltages, is shown in Fig. 4. From the S-parameters the switching capabilities of the device are attained, with the reflection coefficient increasing up to $18 \mathrm{~dB}$, and the transmission coefficient decreasing up to $9 \mathrm{~dB}$, when $V_{g}$ is increasing, as shown in Fig. 4. The switch is considered to be closed for $V_{g} \leq 0 \mathrm{~V}$ and open for $V_{g}>60 \mathrm{~V}$.

To develop an equivalent circuit and to extract the equivalent circuit parameters, resistance $\mathrm{R}$, inductance $\mathrm{L}$, capacitance $\mathrm{C}$, and conductance $\mathrm{G}$ of the switch, the Sparameters were first transformed to $\mathrm{ABCD}$ parameters for a transmission line [12]. The calculated results, shown in Fig. 5, indicate that only the shunt components ( $\mathrm{G}$ and $\mathrm{C}$ ) of the line are affected by the presence of the NWs and only at positive gate voltages. The negative $\mathrm{C}$ at positive $V_{g}$ implies the presence of a shunt inductance, and prevents a standard transmission model from being used.

The analysis of the switch shows that at negative gate voltages the NWs are inactive and have a very high impedance, and therefore do not affect the CPW transmission line. To extract the characteristics of the NWs only, a distributed transmission model, consisting 10 segments, was implemented using Keysight's ADS ${ }^{\mathrm{TM}}$. The number of segments was chosen in order to match the data appropriately. Firstly, the components of the CPW only, were obtained using the S-parameters 


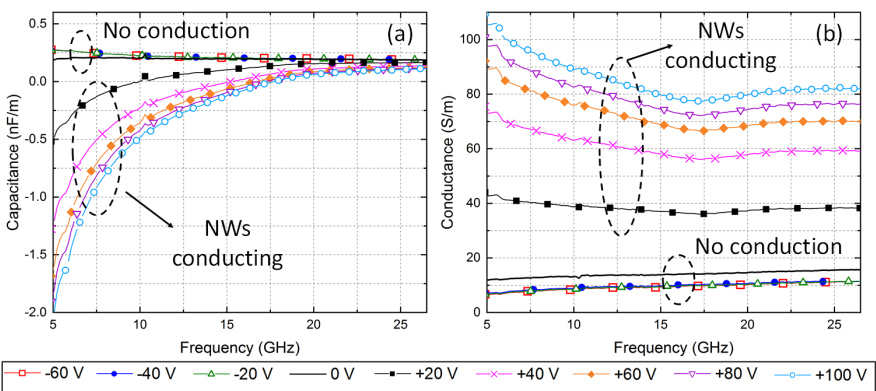

Fig. 5. Calculated (a) capacitance and (b) conductance of the switch obtained from the ABCD parameters.

TABLE I

Circuit Model Parameters

\begin{tabular}{ccccc}
\hline $\boldsymbol{V}_{\boldsymbol{g}}(\mathrm{V})$ & $\boldsymbol{R}_{\boldsymbol{n} \boldsymbol{w}}(\mathrm{M} \boldsymbol{\Omega} / \mathrm{m})$ & $\boldsymbol{L}_{\boldsymbol{n} \boldsymbol{w}}(\boldsymbol{\mu} \mathrm{H} / \mathrm{m})$ & $\boldsymbol{\Delta} \boldsymbol{C}(\mathrm{pF} / \mathrm{m})$ & $\boldsymbol{\Delta} \boldsymbol{G}(\mathrm{S} / \mathrm{m})$ \\
\hline \hline 20 & 7.29 & 381.6 & 21.1 & 26.3 \\
40 & 3.59 & 191.1 & 26.1 & 45.7 \\
60 & 2.78 & 149.6 & 33.9 & 56.2 \\
80 & 2.47 & 133.1 & 34.3 & 61.3 \\
100 & 2.22 & 121.7 & 35.8 & 66.5 \\
\hline
\end{tabular}

at $V_{g}=-60 \mathrm{~V}$ and a distributed model was used to match the data. Fig. 6(a) shows a single segment of the CPW model. In order to match the measured data of the switch at positive gate voltages, a new 10 segment distributed model was used, referred to as switch model, with one of its segments shown in Fig. 6(b). Table I presents the optimised parameter values of the switch model, and Fig. 7 shows the agreement between the two distributed models and the measured data. The two models match the measured data within an error of $8 \%$. The intrinsic characteristics of the nanowires are represented by a resistive $\left(R_{n w}\right)$ and an inductive $\left(L_{n w}\right)$ component, whereas there is a noticeable change in the capacitive $(\Delta C)$ and conductive $(\Delta G)$ components of the CPW. The $\Delta G$ is due to an increased current flow through the substrate, caused by the the current and voltage along the nanofibers, whereas $\Delta C$ is due to the misplacement of NWs within the gaps of the CPW, which were activated under positive $V_{g}$.

\section{CONCLUSION}

This paper described the implementation of an accurate model, representing the high-frequency response of a bottomgated FET switch consisting solution processable InAs nanowires. The model of the device shows good agreement with the measured S-parameters of the switch and enables the investigation of such materials in further microwave applications. The design and fabrication of the switch have also been presented.

\section{REFERENCES}

[1] A. Sahu, P. H. Aaen, A. Lewandowski, M. Shkunov, G. Rigas, P. T. Blanchard, T. M. Wallis, and V. K. Devabhaktuni, "Robust microwave characterization of inkjet-printed coplanar waveguides on flexible substrates," IEEE Trans. Instrum. Meas., vol. 66, no. 12, pp. 3271-3279, Dec. 2017.

[2] L. Yang, A. Rida, R. Vyas, and M. M. Tentzeris, "RFID tag and RF structures on a paper substrate using inkjet-printing technology," IEEE Trans. Microw. Theory Techn., vol. 55, no. 12, pp. 2894-2901, Dec. 2007.

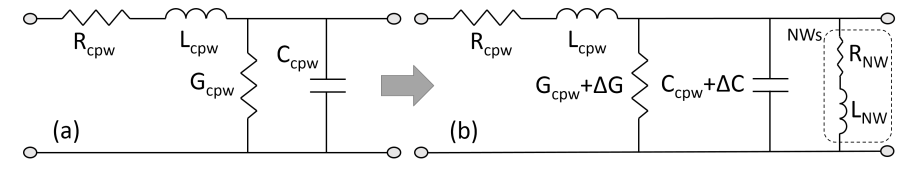

Fig. 6. (a) Single segment of the CPW model, (b) Single segment of the switch model with the NWs being active.
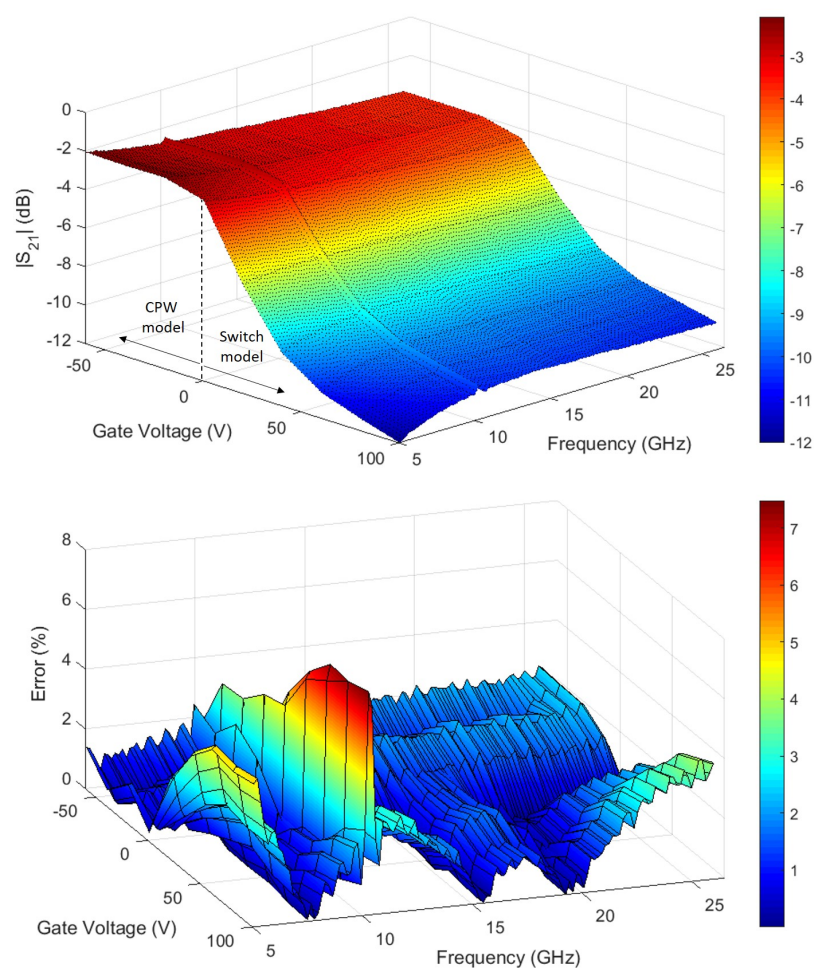

Fig. 7. Simulated $S_{21}$ using equivalent circuit models and error percentage between simulated data and measured data.

[3] G. A. Casula, G. Montisci, and G. Mazzarella, "A wideband pet inkjetprinted antenna for UHF RFID," IEEE Antennas Wireless Propag. Lett., vol. 12, pp. 1400-1403, Oct. 2013.

[4] B. Ando and S. Baglio, "Inkjet-printed sensors: a useful approach for low cost, rapid prototyping," IEEE Instrum. Meas. Mag, vol. 14, no. 5, pp. 36-40, Oct. 2011.

[5] B. Ando, "Inkjet printing: A real opportunity for the next generation of low-cost sensors," IEEE Instrum. Meas. Mag, vol. 16, no. 3, pp. 44-48, Jun. 2013.

[6] G. M. Rebeiz and J. B. Muldavin, "RF mems switches and switch circuits," IEEE Microw. Mag., vol. 2, no. 4, pp. 59-71, Dec. 2001.

[7] Y. Xu, "Post-CMOS and post-MEMS compatible flexible skin technologies: A review," IEEE Sensors J., vol. 13, no. 10, pp. 3962-3975, Oct. 2013.

[8] H. Joyce, J. Wong-Leung, G. Qiang, T. Hoe, and C. Jagadish, "Phase perfection in zinc blende and wurtzite III-V nanowires using basic growth parameters," Nano Lett., vol. 10, no. 3, pp. 908-915, Feb. 2010.

[9] M. Constantinou, G. Rigas, F. Castro, V. Stolojan, K. Hoettges, M. Hughes, E. Adkins, B. Korgel, and M. Shkunov, "Simultaneous tunable selection and self-assembly of Si nanowires from heterogeneous feedstock," ACS Nano, vol. 10, no. 4, pp. 4384-4394, Mar. 2016.

[10] S. Nadar, C. Rolland, J. Lampin, X. Wallart, P. Caroff, and R. Leturcq, "Tunnel junctions in a III-V nanowire by surface engineering," ACS Nano, vol. 8, no. 3, pp. 980-989, Mar. 2015.

[11] R. B. Marks, "A multiline method of network analyzer calibration," IEEE Trans. Microw. Theory Techn., vol. 39, no. 7, pp. 1205-1215, Jul. 1991.

[12] D. Pozar, Microwave Engineering, 4th ed. Wiley, 2011. 